\title{
Fracture analysis of a cooling water pump shaft
}

\author{
Dimitris G. Papageorgiou ${ }^{1,2}$, Kyriakos A. Kovsenoglou ${ }^{1}$, Petros Bournelis $^{3}$ and Carmen \\ Medrea $^{1, *}$ \\ ${ }^{1}$ University of West Attika, Department of Mechanical Engineering, 250 Thivon \& Petrou Ralli Ave. \\ 12244 Egaleo, Greece \\ ${ }^{2}$ Stassinopoulos-Uddeholm, Steel Trading S.A., 20 Athinon Str. 18540 Piraeus, Greece \\ ${ }^{3}$ GasLog LNG Services Ltd., 69 Akti Miaouli 18537 Piraeus, Greece
}

\begin{abstract}
Six shaft failures were encountered in a centrifugal water pump, part of the engine cooling system of a container ship. The last two failed pieces were received for analysis. A detailed study was carried out to determine the cause of the component failures. Historical data was collected, visual inspection was performed and a photographic file was created. The pieces were measured in order to record their general features and a 3-D model was generated. Hardness measurements were carried out. Microstructure was examined by means of light microscopy. A finite element simulation was conducted in order to determine the stress topology and to identify possible critical areas. Chemical analysis was carried out. The shafts were manufactured onboard, from AISI 304 stainless steel. The low hardness of both pieces indicates insufficient mechanical properties. Microstructural examination showed characteristic microstructure of coarse austenite. The presence annealing process derived twins and limited grain-boundary carbide precipitates were verified. The shafts failed due to torsional fatigue. Fracture initiated at the keyway on the propeller side as it was predicted from the finite elements analysis. Material selection material has and poor machining were found to be the main cause of failure. Appropriate recommendations were provided.
\end{abstract}

\section{Introduction}

The global shipping industry is a huge challenge. Millions of people and thousands of products travel from one end of the world to the other through travel or cargo ships. Pumps are one of the most common engineering components of ship's equipment, used for a number of applications; fuel, lubricating oil or water transfusion, as well as, for the hydraulic movement of helms and hoists. Pumps are designed to generate pressure. According to demand, they function in various ranges of temperature, pressure, and power output requirements. A pump configuration may vary, depending on the specific application. They are generally divided into two main categories: dynamic and positive displacement. In terms of operation method, pumps are classified as rotary, reciprocating, centrifugal and axial.

The hydraulic pumps belong to the dynamic type and create the pressure using liquid velocity. Centrifugal pumps have an extensive range of applications, from goods processing to circulation of raw material. It is a device employed to move fluids from one place to 
another by increasing their pressure using the centrifugal force that accelerates the liquid being handled. One of the most common types is the water pump, mostly used were high flow rates and moderate head increases are required.

In shipping, the water pump is considered the heart of the engine's cooling system. It circulates uninterruptedly the water throughout the entire cooling circuit and regulates the coolant flow rate [1]. Its working principle is based on the impeller rotation that causes the water to flow towards the periphery due to the centrifugal force generated. Suction from the impeller draw water into pump (inlet) and the water is discharged on other casting side.

A centrifugal water pump comprises of the casing, the bearing housing, the pump shaft and the impeller [2]. The shaft supports the rotating components and transmits motion by rotary movement [3]. This pump component operates under hostile temperature, stresses and environmental conditions [4]. It can also be subjected to vibrations [5]. Consequently, it is not unexpected to suffer from degradation. Shaft damage is associated with corrosion and mechanical degradation, usually in the form of fatigue failure [6]. Fatigue is caused under a substantially lower stress value than the materials' yield strength. In many cases, corrosion precedes fatigue failure, reduces the fatigue resistance of a material - accelerating the failure - and dramatically decreases the component's working life [7].

This paper presents the failure analysis of two shafts, part of a centrifugal water pump. Shaft damage entrains operational changes that reduce pump efficiency or cause its breakdown. The water flow will be reduced or interrupted. This will definitely affect negatively the output of the cooling system, either partially or completely. Indirectly, this will affect harmfully other parts of the cooling system by slowing them down or causing them to perform undesirably. Implicitly, any shaft failure will raise downtime, material and labour costs and therefore, must be avoided.

\section{Experimental}

Historical data of the failed pieces was collected. All the available information regarding their in-service conditions was gathered. Non-destructive examination was performed, including visual inspection as well as inspection under stereoscope. The general features of the surface were evaluated. 3-D models of the pieces were generated using Inventor CAD software. The examination was focused on the fracture areas. An extensive photographic file was created. The status of the received pieces was affected by improper storage, with wide surface areas corroded. After the visual inspection, corrosion products were removed by means of sandblasting. The shafts were further cut into experimental samples, and representative specimens were prepared and examined. Rockwell hardness was measured circumferentially on the surface on several axial locations of both shafts. Chemical analysis was performed using optical transmition spectroscopy. The results were run against standards' database to examine whether the selected material meets the suitable specifications. The metallographic examination was conducted by light microscopy. The shaft microstructure, the grain size, and other details were investigated. Finally, a finite element simulation was conducted in order to determine critical location of stresses using SolidWorks software.

\section{Results and discussions}

A container ship uses a centrifugal water pump to cool the engine (Fig. 1). The pump failed repeatedly. Six shafts, part of the cooling system, broke down in a period of three months. The shafts were manufactured onboard. Most probably, no heat treatment was carried out after machining. Compulsory repairs and shaft replacement took place in open sea. The last 
two failed pieces were received for analysis. The first one (N1) operated for only 24 hours after its placemen. The second (N2) operated for 72 hours.

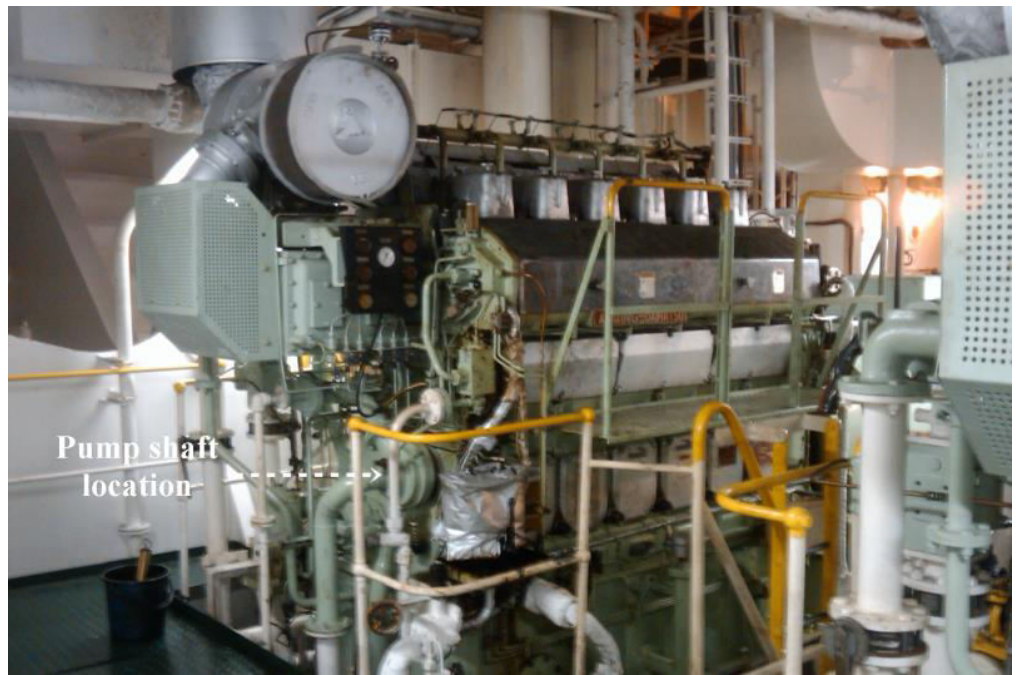

Fig. 1. (a) General aspect of the ships engine. The arrow indicates the pump shaft location.

According to the ship engineers, the maintenance procedure of the pump was carried out regularly and in accordance with the engine's manufacturer specifications. The pump was regularly checked for any degradation signs. Improper roller bearings or waterproofing rings were replaced on time. Coolant maintenance was regular, including addition of anticorrosion agents. Notably, unusual noise or excessive vibrations, a fact that could raise suspicion of malfunction of the system, were not detected during pump operation.

Shafts are $315 \mathrm{~mm}$ long and have a circular cross-section. They are divided in eight sub sections of unequal diameters (varying from $18 \mathrm{~mm}$ to $42 \mathrm{~mm}$ ) and lengths (Fig. 2).

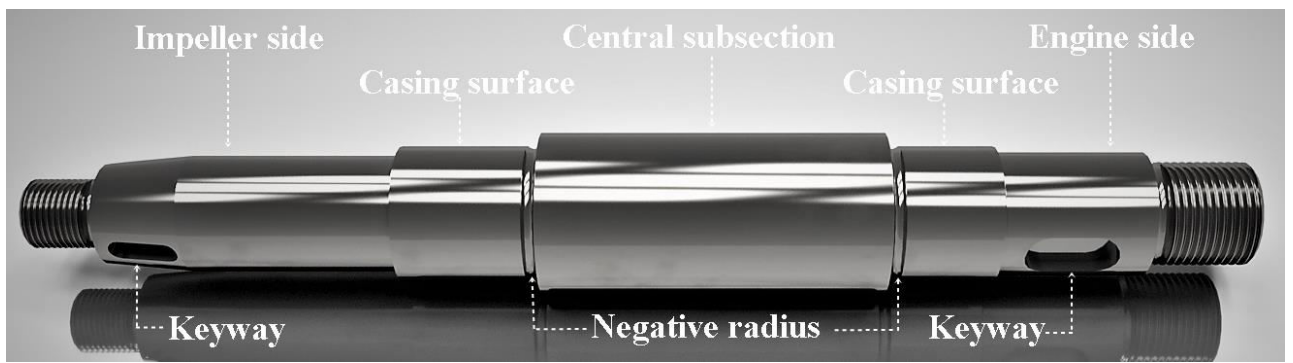

Fig. 2. The 3D shaft geometry generated using Inventor program. The model was created based on the dimensional measurements of the failed pieces.

Both axes have failed on the side securing the pump impeller (Fig. 3a). The first piece broke into two parts. In the case of the second shaft, small fragments were detached from the body. In both cases, the keyway has been overwhelmed.

Visual inspection revealed poor machining procedure. The general feature indicates that the shafts were manufactured in conventional lathe. On both sides of the central subsection, areas of negative radii on the chamfer were machined in order to fix a bearing. From a design point of view, this is a poor choice since the specific area becomes a stress raiser.

The first shaft is in a worse condition. The bottom of the keyway has rough surface in several spots and appears deformed locally. Welding overlap (point I in Fig. 3b) and welding undercut (point II in Fig. 3b) are apparent around the base of the thread. 
Furthermore, sputters are distributed on the surrounding area. These clues suggest welding intervention. The casing bearing surface looks as if it had been polished with grinding paper, either for dimensional adjustment (due to tight tolerance), or to improve surface quality. On the central subsection, specific striations suggest rough fine processing, probably due to excessive feed rate of the cutting tool during manufacturing. Characteristic milling marks on the second shaft indicate of poor-detail fine machining (Fig. 3c).

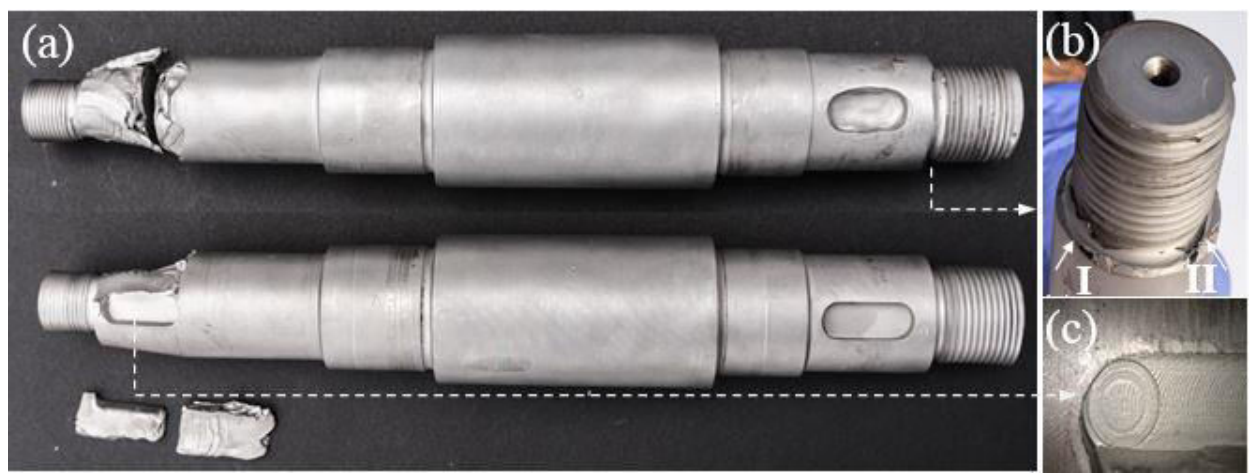

Fig. 3. (a) General view of the failed shafts, after sandblasting, (b) Welding overlap and undercut around the thread base of the first piece, (c) Milling marks on the keyway bottom of the second piece.

Both shafts broke down under torsion. The first piece (N1) had been overloaded by a torsional stress. The fracture surface is typical of fatigue failure, comprised of thee specific zones. It started at the edge of the keyway (point I Fig. 4a,c). Cracks propagated around the shaft's circumference (Fig. 4b). They grew through almost half of the radius following specific progression lines, leaving a typical feature on the circumference, visible to the naked eye (torsional marks). Subsequently, the fracture surface developed on the cross section of the piece. This area is shining due to the wear of the surfaces of the two fractured parts, indicating that the failure was not immediately detected and the pump continued to work after cracking. That may have caused unpredicted effect on the other pump components. Fracture ends at the side nearly opposite of its origin, in a comparatively rough detachment zone where the final detachment occurred (Point II on Fig.4c). The large size of the fatigue zone, in comparison with the small final detachment zone is an indication of material's low fatigue resistance.
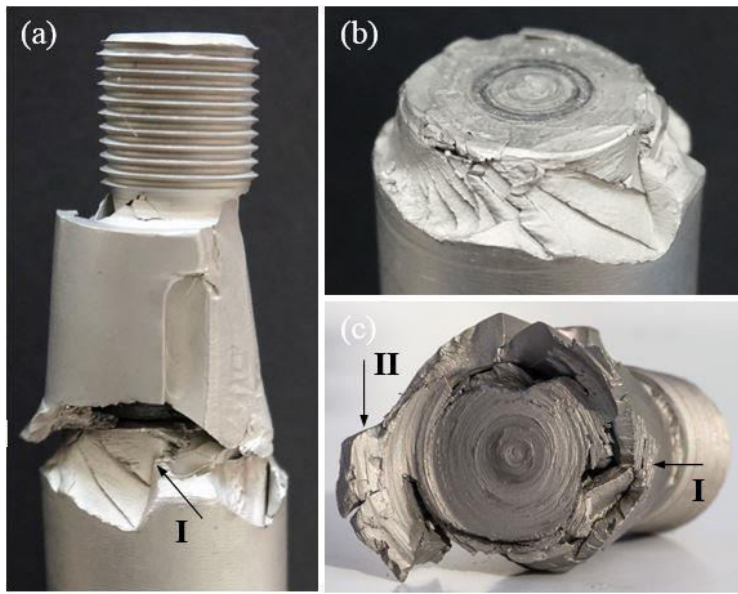

Fig. 4. Details of the first shaft; (a) Crack originate at the edge of the keyway, (b) Fracture overview showing torsion marks developed around shaft's circumference, (c) General overview of the fragment fracture surface. 
The second shaft (N2) failed near the securing impeller site (Fig. 5a). At low magnification, under stereoscope, the fracture front was located at the side of the keyway (narrows on Fig. 5b). Crack developed under torsion stresses in fatigue mode, living thumbnail pattern [8] on the fracture surface (narrow on Fig. 5c). Two fragments detached after fracture. A small fatigue zone in comparison with the largest detachment zone indicates a high fatigue stress
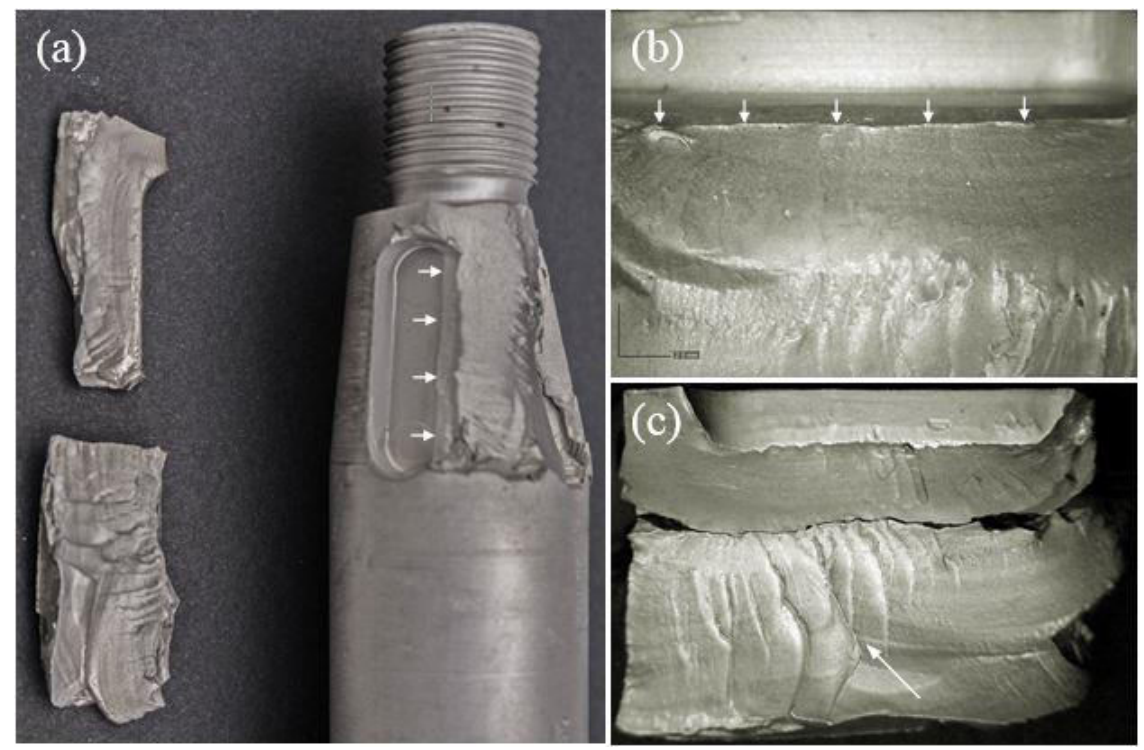

Fig. 5. Details of the second shaft; (a) General aspect of the fracture surfaces, (b) Fracture overview showing the origin of the crack (stereoscope image), (c) Thumbnail pattern observed on the fracture surface of the fragment (stereoscope image).

Chemical analysis indicated that the two shafts have been manufactured of AISI 304 steel. Table 1 gives the chemical composition of the pieces compared against the standard composition [9]. 304 is a chromium-nickel austenitic stainless steel. It is a corrosion resistant material, simple to clean, easy to manufacture, with excellent forming and welding characteristics [10]. It is the most commercial steel grade of its group, offered in a large variety of forms and finishes on a number of products, readily available in every machinery shop around the world [11]. Is the best choice for the manufacturing of a variety of shafts, marine equipment and fasteners, but is not recommend for pump shaft making. AISI 304 is readily available onboard; consequently it is usually employed in case of emergency. It is however inadequate for specific application due to limited mechanical properties and absence of fatigue resistance limit. Pump shaft industry propose a large range of stainless steel grades considerably superior, able to meet particular requirements in shipping applications $[12,13]$.

Table 1. Chemical composition of the shafts compared to standard composition [9].

\begin{tabular}{|c|c|c|c|c|}
\hline \multirow{2}{*}{} & \multicolumn{4}{|c|}{ Chemical elements Wt. \% } \\
\cline { 2 - 5 } & $\mathrm{C}$ & $\mathrm{Mn}$ & $\mathrm{Cr}$ & $\mathrm{Ni}$ \\
\hline First shaft & - & 1.04 & 18.00 & 8.22 \\
\hline Second shaft & - & 1.27 & 18.45 & 7.83 \\
\hline Nominal composition & max. 0,08 & max. 2 & $18-20$ & $8-11$ \\
\hline
\end{tabular}


The first shaft exhibited uniform hardness of 91- 93 HRB and the second 87- 93 HRB, respectively. In both situations, the low hardness values indicate inability to withstand the required loads.

Both shafts present characteristic microstructure of stainless steels consisted of coarse austenite. Twins can be observed due to annealing process. Limited grain boundary carbide precipitates were found (Fig. 6).
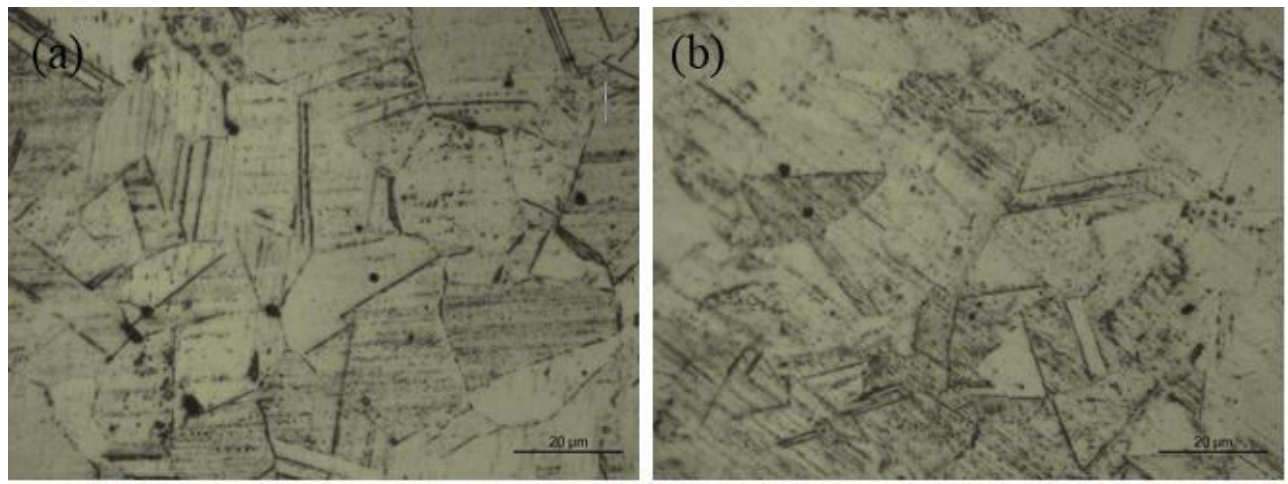

Fig. 6. Optical microstructure; (a) First shaft, (b) Second shaft.

Data obtained using 3D finite element stress analysis pinpointed the critical area of stress concentration (maximum equivalent stress). The specific area is situated on the base of the keyway on the propeller site (Fig. 7a). It is subjected to high torsion stresses undergoing high strain. Being the most susceptible to fail, it seems the most probable point of crack origin (Fig. 7b). Figure 7c shows that the fracture occurred on the predicted critical zone.
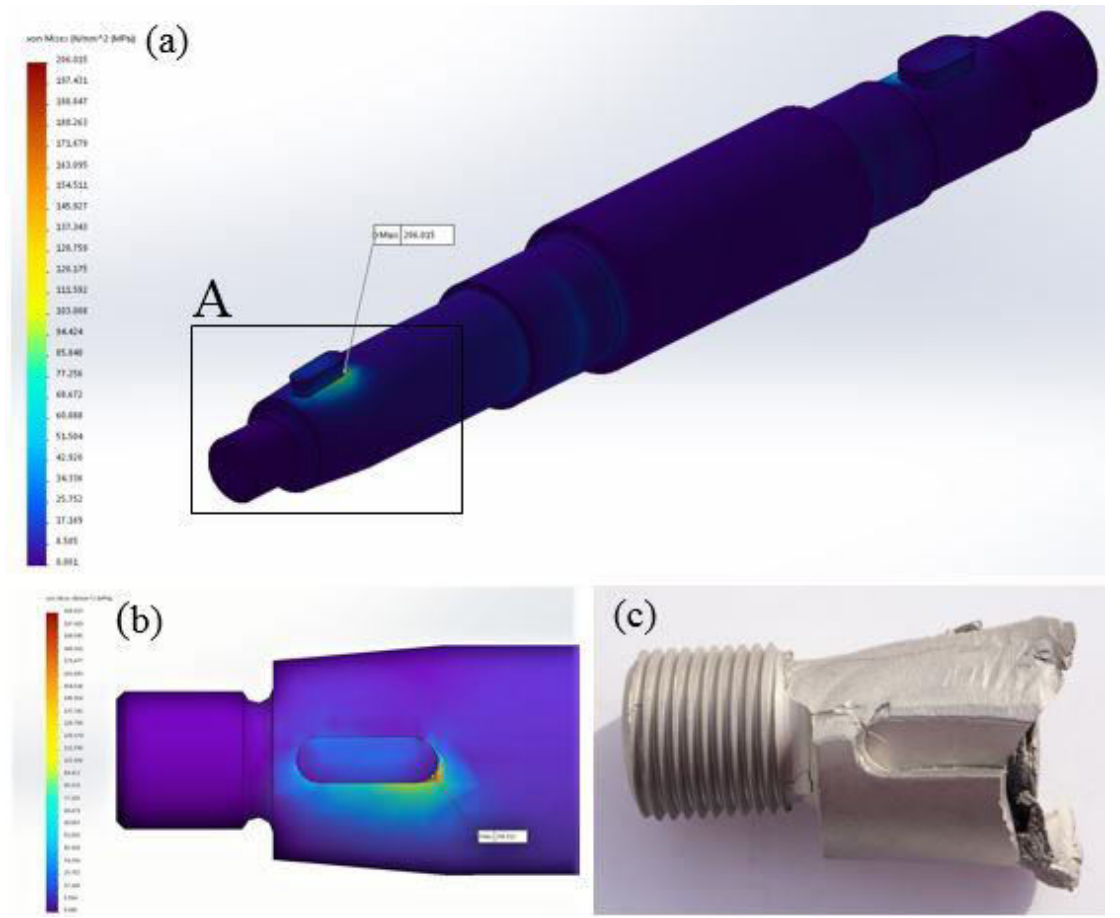

Fig.7. 3D finite elements stress analysis on shaft N1 (performed on SolidWorks); (a) Overview of the model of the shaft. The keys are modelled fitted on the respected keyways, (b) Zoom of the area A, depicting the critical area of stress, (c) Actual photograph of the fragment, shown for comparison. 


\section{Conclusions}

The two shafts failed due to torsional fatigue. In fact, the fracture mechanism is torsionspecific. Fracture initiated at the keyway on the propeller side. This coincides with the critical zone predicted as per finite elements analysis. The selection of inappropriate material has been identified as the main cause of failure. Events were dramatically accelerated due to poor machining.

Similar issues should be faced with a more direct approach as imposed from their immediate nature. The ship personnel responsible for maintenance should seek advice and apply the recommendations available on the pump-shaft industry, regarding the material selection and the quality of machining. Provision of adequate supplies of appropriate steel grades, available on board, are vital in order to handle emergency situations.

From an economic and productive point of view, the consequences can be quantified taking into account the overall cost of repairs, part replacements as well as the shutdown time required to carry them out.

Authors would like to acknowledge Tyros Panagiotis and Kouklakis Filippos, undergraduate students of the department of Mechanical Engineering of the University of West Attika, for their contribution on 3D modelling and finite elements analysis.

\section{References}

1. A. Hamid, S. Nugroho, G. D. Haryadi, Khaeroman, MATEC Web of Conferences 159, 02027 (2018)

2. S. Shiels, "Optimizing centrifugal pump operation," World pumps, vol. 2001, pp. 3539, 2001.

3. R. Celin, F. Tehovnik, F. Vodopivec, B. Zuzek, MTAEC9, 47(2), 253 (2013)

4. W. Muhammad, K. M. Deen, J. Fail. Anal. and Preven. 10, 161 (2010)

5. M. M. Onari, V. G. Arzani, Pump \& Turbo, pumpturbo.tamu.edu (2014)

6. G. Das, A.N.Sinha, S.K. Mishra, D.K. Bhattacharya, Eng. Fail. Anal. 6, 267 (1999).

7. F. Berndt, A. van Bennekom, Eng. Fail. Anal. 8, 135 (2001)

8. K.A.Esaklul, Handbook of Case Histories in Failure Analysis 2 (ASM International, Ohio, 1993)

9. Verlag Stahlschlussel Wegst GmbH (2007), Stahlschlussel, 21th edition (Version 5.0) "Software", Verlag Stahlschlussel Wegst GmbH, Marbach.

10. Lenntech, Steel fact, https://www.lenntech.com/stainless-steel-304.html

11. Aerospace Specification Metals, AISI Type 304 Stainless Steel, http://asm.matweb.com/search/SpecificMaterial.asp?bassnum=mq304a

12. Mindiamart, Stainless steel shaft, https://dir.indiamart.com/impcat/stainless-steelshafts.html

13. Best, Stainless \& Alloys, Pump shaft, https://www.beststainless.com/pump-shaft.html 